\title{
Habilidades de argumentación escrita en la universidad
}

\author{
(Written Argumentation Skills of University Students)
}

\author{
LiNALOe LeÓN BARo (iD, Universidad de Sonora, Sonora, México
}

\author{
Volumen 5, número 1 \\ Enero-junio de 2020 \\ p. $1-31$
}

Este número se publicó el 14 de enero de 2020

Artículo recibido: 19 de septiembre de 2018

Artículo aprobado: 10 de diciembre de 2019

ISSN: 2448-5942 doi: https://doi.org/10.36799/el.v5i1.102

\section{Citar este artículo como:}

León Baro, L. (2019). Habilidades de argumentación escrita en la universidad. Estudios גambda. Teoría y práctica y de la didáctica en Lengua y Literatura. , 5(1), 1-31. https://doi.org/10.36799/el.v5i1.102

Derechos de autor: El autor o autores conservan en todo momento sus derechos morales y patrimoniales sobre la obra; la obra no se puede alterar, transformar o ampliar; siempre debe reconocerse la autoría del documento referido. Ninguna de las modalidades de los documentos publicados en Estudios $\lambda$ ambda. Teoría y práctica de la didáctica en lengua y literatura tienen fines comerciales de naturaleza alguna.

Los contenidos de este artículo están bajo una licencia de Creative Commons Atribución No Comercial- Sin Derivadas 4.0 Internacional

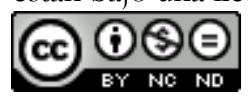




\title{
Habilidades de argumentación escrita en la universidad
}

\author{
Written Argumentation Skills of University Students
}

LINALOE LEÓN BARO 1

\begin{abstract}
RESUMEN
En este trabajo de investigación se concentran los resultados obtenidos hasta el momento con respecto al análisis de una parte del corpus total de tesis doctoral; específicamente, se trata del análisis descriptivo de 28 textos argumentativos escritos por un grupo de estudiantes de primer año de la Licenciatura en Lingüística de la Universidad de Sonora, quienes participaron en dos sesiones de trabajo con diversas actividades -como lectura, discusión oral y exposición de información-, que dieron como resultado dos versiones de textos por cada alumno. El objetivo es conocer cómo los estudiantes construyen los argumentos con los que defienden su punto de vista sobre un tema polémico general, además de revisar los cambios que pudieran presentarse entre cada una de las versiones de los escritos elaborados en las dos fases de escritura. Para ello, los textos fueron estudiados a partir de una propuesta de análisis que tiene su base en el marco pragma-dialéctico de la argumentación, la cual consiste en trasladar los textos a una versión gráfica, de forma que puede analizarse la estructura general de los textos al hacer visibles los distintos elementos argumentativos que utilizaron los estudiantes para conformar la defensa de su punto de vista. Los resultados mostraron que, en los textos de la primera sesión de trabajo, los estudiantes usaron una cantidad moderada de elementos argumentativos totales, pero la diversidad de tipos de recursos se mostró limitada. Por otra parte, en los escritos resultantes de la segunda sesión de trabajo se registró un aumento general tanto en la cantidad de elementos totales usados como en la diversidad de éstos, lo cual se reflejó principalmente en la inclusión de componentes que implican cierto grado de conciencia sobre la existencia de una audiencia crítica que puede cuestionar su postura personal.
\end{abstract}

PALABRAS CLAVE: escritura, habilidad, discurso, razonamiento, opinión, estudiantes universitarios.

\begin{abstract}
In this article, we present the results of a research work done in a doctoral thesis. In this case we concentrate in the results obtained so far with respect to the analysis of a part of the total corpus of the doctoral thesis; specifically, it is the descriptive analysis of 28 argumentative texts written by a group of first-year students of Linguistics Program at the University of Sonora, who participated in two work sessions with various activities -such as reading, oral discussion and exposition of information-, which resulted in two versions of a text for each student. The objective of the research is to describe how the students elaborate the arguments to defend their points of view over a general controversial topic, as well as to analyze the changes that appeared between each version of the texts elaborated in the two phases of the composition process. Methodologically, the texts were first analyzed from a pragmatic-dialectical framework of argumentation, which consists in transferring the texts to a graphic version, so that the general structure of the texts can be then analyzed by making visible the different argumentative elements that the students used to construct the defense of their points of view. The results showed that in the texts of the first working session, students used a moderate amount of total argumentative elements, but the diversity of types was limited. On the other hand, in the texts resulting in the second working session there was a general increase, both in the amount of total elements used and in the diversity of these, which was reflected mainly in the inclusion of components that imply a certain degree of awareness about the existence of a critical audience that may question their opinion.
\end{abstract}

KEYWORDS: writing, skills, speech, reasoning, university students

${ }^{1}$ Maestría en Lingüística. Universidad de Sonora. linaloeleba@gmail.com

Artículo recibido: 19 de septiembre de 2018

Artículo aprobado: 10 de diciembre de 2019

Linaloe León Baro doi: https://doi.org/10.36799/el.v5i1.102 Volumen 5, Número 1, Año 2020, ISSN: 2448-5942 


\section{INTRODUCCIÓN}

La argumentación es un tipo de discurso que está habitualmente presente en la vida cotidiana, ya que las diferencias de opinión surgen frecuentemente en los distintos ámbitos de la vida pública, ya sea a nivel personal, laboral, académico, etcétera. Es una actividad discursiva que conlleva un proceso de razonamiento en el que una persona expone y defiende su opinión sobre un tema, oralmente o por escrito, con la intención de convencer a una audiencia de que acepte o adopte su postura, por lo que ha de ofrecer razones que muestren su aceptabilidad (Calsamiglia y Tusón, 2004; Felton y Herko, 2004; Rodríguez, 2004; Van Eemeren et al, 2004, 2006).

Uno de los entornos en el que se hace un uso frecuente de este tipo de discurso es el educativo, ya que constantemente se propicia en el aula la discusión crítica sobre algún tema; en esos debate orales, los estudiantes pueden mostrarse entusiasmados al tener que elaborar y presentar sus argumentos para defender su idea, sin embargo, al momento de tener que presentar una defensa por escrito los argumentos tienden a ser menos detallados, por lo que puede perderse mucha de la fuerza y elocuencia que tienen sus argumentos en la discusión oral (Felton y Herko, 2004).

Esto es porque la lengua escrita es una manifestación del lenguaje que involucra convenciones lingüísticas y mecanismos cognitivos distintos de los que caracterizan a la lengua oral, a pesar de que ambas sean proyecciones de un mismo sistema. En una conversación, por ejemplo, la planeación y organización de lo que se quiere comunicar ocurre al mismo tiempo que se produce el habla, además, tanto el oyente como el hablante se encuentran en interacción directa, por lo que puede haber retroalimentación, resolución de dudas y reformulaciones al tiempo que se conversa. En cambio, la formulación del discurso escrito implica un proceso complejo distinto en el que se involucran distintos mecanismos cognitivos y estrategias que guían al escritor durante la producción textual. Este proceso va desde la concepción del tema, la planeación, generación y organización de ideas hasta la redacción, revisión y edición del escrito. Además, a pesar de que la interacción a través de la lengua escrita no se da en tiempo real, quien escribe debe estar consciente de que su texto será leído por alguien y por lo tanto debe ajustar su composición a esa audiencia, haciendo que su escrito sea suficientemente coherente y claro, de modo que el lector logre interpretar el mensaje con mayor facilidad.

Linaloe León Baro doi: https://doi.org/10.36799/el.v5i1.102 Volumen 5, Número 1, Año 2020, ISSN: 2448-5942 
Particularmente en la educación de nivel superior, la lengua escrita juega un papel importante durante la formación académica de los estudiantes, al ser un medio básico para la obtención de información y apropiación de los contenidos durante la formación académica, por lo que necesitan desarrollar estrategias que les permitan conducirse de forma eficiente durante las actividades de lectura y escritura. Pero, además de ser capaces de poder regular con eficiencia sus estrategias de producción textual, en la argumentación escrita deben lograr asumir y exponer un punto de vista sobre un tema y justificarlo, anticipar la postura del lector, considerar posiciones alternativas y refutar ideas opuestas cuando sea necesario.

Entonces, debido a las demandas intelectuales implicadas en el proceso de escritura y las particularidades del discurso argumentativo escrito, es de esperarse que los estudiantes tengan dificultades cuando se enfrentan a la tarea de producir textos argumentativos. Así pues, este trabajo surge del interés por saber más sobre las habilidades de argumentación escrita con las que cuentan los estudiantes universitarios. Es un estudio cuyo propósito es conocer cómo los estudiantes construyen sus argumentos para defender un punto de vista sobre un asunto polémico de dominio general; para esto, se trabajó un corpus de textos escritos por estudiantes de primer semestre de licenciatura de la Universidad de Sonora que fue estudiado a partir de una propuesta de análisis que tiene sus bases en el marco pragma-dialéctico de la argumentación.

\section{ANTECEDENTES DE INVESTIGACIÓN}

El interés por la modalidad escrita de la lengua en el ámbito educativo ha fomentado la realización de estudios que buscan obtener más conocimiento sobre el desempeño que muestran los estudiantes de diferentes niveles educativos al enfrentarse a actividades de escritura argumentativa. En general, las investigaciones tienden a evidenciar que a los estudiantes se les dificulta alcanzar un desempeño satisfactorio en este tipo de actividades.

Un primer ejemplo dentro del ámbito de la argumentación escrita es el realizado por Chase (2011) de la Universidad de Columbia. Esta investigación se enfoca en las habilidades de escritura argumentativa de una muestra de estudiantes de un Community College que contaban con un historial de bajo rendimiento académico; para el análisis de la calidad textual global, la autora se centró en la inclusión de elementos argumentativos en los ensayos, la coherencia y la cohesión. 
Además, analizó el posible impacto de algunas variables demográficas en los ensayos, como la habilidad de lectura, conocimiento e interés científico, género y lengua nativa de los alumnos.

La muestra del estudio de Chase estuvo conformada por un total de 112 estudiantes que completaron varias actividades, incluida una tarea de escritura. Ésta última consistió en desarrollar un ensayo argumentativo sobre un tópico controversial. Para evaluar el desempeño según la presencia o ausencia de elementos argumentativos, los ensayos fueron traspasados a una forma gráfica, de modo que se apreciaran con claridad los elementos del texto y sus relaciones; posteriormente se aplicaron algunas escalas de evaluación para medir la calidad de los ensayos y el desempeño en los aspectos de coherencia y cohesión.

En sus resultados encontró que los estudiantes escribieron ensayos que en promedio estaban parcialmente desarrollados, incluyeron una cantidad moderada de elementos funcionales, la coherencia estaba desarrollada parcialmente y mostraron un uso mínimo de enlaces cohesivos. Además, tanto los componentes del ensayo y las características demográficas del escritor estaban significativamente asociados con la calidad de los ensayos escritos por la muestra de estudiantes. Específicamente, notaron que la coherencia es un indicador fuerte de la calidad de los textos, pero no tanto la inclusión de elementos funcionales de la argumentación o el uso de enlaces cohesivos.

En el contexto de la Educación Superior en México aún es escasa la realización y socialización de investigaciones sobre el desempeño de los estudiantes de Nivel Superior en la argumentación escrita, sin embargo, es posible encontrar algunos estudios dentro de este campo. Uno de ellos fue realizado por Bañalez Faz, et al (2015), en el que se puso en práctica una intervención didáctica con 25 estudiantes de primer año de la Licenciatura en Lingüística Aplicada; con una duración de 40 horas de trabajo encaminado a cumplir con la instrucción que les fue asignada en un principio: elaborar un ensayo argumentativo sobre un tema discutible dentro su campo de formación académica.

Una parte de las tareas realizadas estuvieron dentro de una primera secuencia didáctica centrada en las labores de andamiaje por parte de dos profesores para apoyar el análisis de cinco lecturas que les fueron proporcionadas a los alumnos como fuentes de información sobre el tema sujeto a discusión; en esta fase realizaron tareas de abstracción de ideas principales, de análisis crítico y debates grupales que sirvieron para comprender el tema al mismo tiempo que Linaloe León Baro doi: https://doi.org/10.36799/el.v5i1.102 Volumen 5, Número 1, Año 2020, ISSN: 2448-5942 
advertían aspectos importantes sobre la construcción de argumentos. Después, en una segunda secuencia se instruyó a los estudiantes sobre la argumentación y elaboración de ensayos; aquí tuvieron la oportunidad de elaborar su texto en las mismas instalaciones escolares, de modo que contaron con la guía directa de sus profesores para solucionar dificultades de contenido y forma durante su proceso de escritura.

Al finalizar ambas secuencias, tanto estudiantes como profesores respondieron una serie de preguntas sobre sus experiencias, con el fin de analizar el impacto de la intervención en los procesos de enseñanza y aprendizaje de la argumentación escrita disciplinar. Los resultados de su exploración dieron muestra de los beneficios y complicaciones que encontraron los participantes durante la intervención; y aunque no se realizó un análisis de calidad de los textos, los autores rescatan el valor de las reflexiones con respecto al diseño de estrategias didácticas que favorezcan el desarrollo de las habilidades argumentativas de los universitarios.

Otro estudio fue realizado por Castro y Sánchez (2013), cuyo objetivo es conocer los recursos lingüísticos que utilizan los estudiantes universitarios para posicionarse discursivamente en un texto escolar de opinión. Su corpus contó con 40 ensayos académicos escritos por estudiantes de licenciaturas en Lenguas modernas aplicadas; esos escritos fueron analizados a partir de tres aspectos: estructura del texto, inserción y manejo de voces, y construcción de perspectiva.

En sus resultados encontraron que los escritos siguieron la misma estructura básica de introducción, desarrollo y conclusión; pero que hubo variaciones en la inclusión de otros elementos como el título, epígrafe y la utilización de citas o referencias a lo largo de la argumentación, igualmente en la integración de notas y de la bibliografía consultada. En cuanto al contenido, constataron que, a pesar de ser textos de opinión, fueron mayormente expositivos, pues se limitaron a presentar un recuento de los datos relevantes de sus fuentes de información, sin realizar un trabajo de análisis que les permita cuestionar la información para aportar evaluaciones personales.

\section{LA ARGUMENTACIÓN ESCRITA EN LA UNIVERSIDAD}

Linaloe León Baro doi: https://doi.org/10.36799/el.v5i1.102 Volumen 5, Número 1, Año 2020, ISSN: 2448-5942 
La escritura argumentativa es una habilidad fundamental durante la formación académica de los estudiantes de nivel superior y su estudio se ubica dentro del amplio marco de la Alfabetización Académica (Academic Literacy), que se define como un conjunto de ideas y estrategias que se necesitan para participar en la cultura discursiva de las disciplinas científicas, y también en las actividades de producción y análisis de textos necesarios para aprender y desarrollar la exposición de nuevos conocimientos en el ámbito académico universitario. Es decir, representa un proceso que sirve para adentrarse en la comunidad científica elegida por el estudiante, a través de la apropiación de los patrones de razonamiento discursivo que se manejan en ella (Carlino, 2005, 2003).

Actualmente, son aún escasas las investigaciones que se realizan en México con respecto a las habilidades generales de lectoescritura en el Nivel Superior, pero se mantiene constante la socialización de estudios centrados en el desarrollo de estas competencias en la Educación Básica. Esto podría deberse a que en algunos entornos se tiene la idea de que el aprendizaje de la escritura se concluye al finalizar los estudios previos a la educación superior y que la adquisición de las habilidades necesarias se completa en algún momento de la formación académica. Sin embargo, esas ideas son cuestionables, ya que la diversidad de temas, tipos de textos, propósitos de la escritura, audiencias, reflexiones y contextos representan nuevos retos para quienes se enfrentan inicialmente a esta tarea y exigen que se siga aprendiendo a escribir (Carlino, 2003, 2005).

Así pues, el planteamiento principal del que parte este trabajo es la necesidad de realizar más estudios sobre el desempeño de los estudiantes universitarios con respecto a la argumentación escrita, ya que las investigaciones consultadas indican que las habilidades de escritura con las que cuentan los jóvenes son insuficientes para desenvolverse satisfactoriamente en diversas actividades de producción textual. Una de las razones que podría llevar a este problema es la falta de énfasis y práctica que se hace sobre este tipo de actividades en el sistema educativo del país, en el que siguen predominando los métodos tradicionales para la enseñanza de la escritura. Estos enfoques tienden a reducir esta actividad al buen manejo de las normas formales de la lengua, dejando de lado el desarrollo de habilidades cognitivas y metacognitivas que le permitan al estudiante tener conciencia de su propio proceso de escritura, de modo que logren monitorear y regular las estrategias que requieren durante la producción textual y que, además, puedan comprender los distintos tipos de textos, sus funciones y propósitos de la 
escritura. Entonces, conocer las dificultades que pueden presentar los alumnos cuando se enfrentan a la tarea de escribir ensayos argumentativos, serviría como base para ayudarlos a mejorar sus habilidades y convertirlos en estudiantes con mayor sentido crítico y en mejores participantes de la práctica de la escritura argumentativa.

En esta línea, el presente trabajo representa un reporte parcial que resulta de los avances de tesis doctoral, que se enmarca dentro del área y la problemática de la alfabetización académica. Se trata de un estudio que busca conocer más acerca de las habilidades de argumentación escrita por parte de estudiantes universitarios, a partir del análisis estructural de su discurso argumentativo escrito. La propuesta de estudio surgió del interés y la necesidad de ampliar el alcance de las investigaciones mexicanas sobre las habilidades de escritura en el nivel superior, específicamente, de la escritura argumentativa. La motivación particular para su realización es un previo trabajo propio de investigación, en el que se analizó la estructura de un corpus de 20 textos argumentativos escritos por alumnos de primer año de licenciatura.

Dicho estudio mostró que los estudiantes sí tenían idea de lo que significa argumentar y de la importancia de defender una opinión, sin embargo, sus textos resultaron poco desarrollados, ya que la estructura de sus argumentos por lo general estuvo compuesta tan sólo por la expresión del punto de vista y una cantidad limitada de razones para defenderlo, haciendo un uso escaso de otros recursos de argumentación como puntos de vista alternativos, contraargumentos y refutaciones, que son elementos a los que podrían recurrir para construir textos argumentativos más complejos y sólidos. Además, al discutir los resultados obtenidos, se identificaron algunas limitaciones en ciertas fases del estudio que podrían haber influido en la poca complejidad de los textos del corpus; una de ellas fue el corto tiempo que tuvieron los participantes para realizar la actividad de escritura, ya que sólo se contó con una sesión de un máximo de 50 minutos, en los que debieron leer una nota informativa sobre el tema a discutir para después elaborar su propio texto. Por estas razones, se propuso continuar el estudio en la misma línea, pero con reformulaciones en los objetivos y metodología, con la finalidad de analizar no sólo la estructura de los argumentos, sino también los cambios que podrían presentarse al elaborar los textos en varias etapas.

\section{OBJETIVO}

Linaloe León Baro doi: https://doi.org/10.36799/el.v5i1.102 Volumen 5, Número 1, Año 2020, ISSN: 2448-5942 
Estudiar un corpus de textos argumentativos elaborados por estudiantes universitarios en dos sesiones de escritura con el fin de conocer su desempeño con respecto a este tipo de discurso; esto a través de un análisis de los elementos argumentativos con los que construyen sus textos, lo que permitirá aportar una valoración de los mismos. Además, se busca analizar las diferencias que pudieran presentarse entre las versiones de los escritos elaborados por los estudiantes en dos distintas sesiones de escritura y siguiendo diversas actividades.

\section{MARCO TEÓRICO-CONCEPTUAL}

Al realizar estudios sobre la habilidad de escritura en el contexto educativo, es útil hacer una revisión sobre las ideas que giran en torno a la enseñanza y desarrollo de esta habilidad del lenguaje y de algunos de los cambios que se han generado con el tiempo en cuanto a la concepción de esta habilidad como parte de la formación académica, esto permite comprender mejor el contexto actual del ámbito en el que se enmarca este trabajo. También es importante conocer las complejidades que conlleva la composición textual, ya que es una actividad que involucra un conjunto de diversos mecanismos cognitivos que guían el proceso de escritura. Así pues, este apartado contiene una exposición general sobre algunos cambios que han surgido en la manera de concebir o conceptualizar la enseñanza de la escritura y al funcionamiento del proceso de escritura.

Posteriormente, dado que el interés central de este trabajo radica en el desarrollo de las habilidades de escritura argumentativa, se presenta también información referente a la argumentación y a algunas propuestas que han surgido a lo largo del tiempo para explicar y analizar las particularidades de este género discursivo; se presta especial atención a propuestas teóricas como el modelo de Toulmin y el marco pragma-dialéctico de la argumentación, que han servido como base de muchas investigaciones actuales sobre la argumentación desde distintas disciplinas, incluida el área de la alfabetización académica.

\section{CONCEPTO Y PRINCIPIOS DE LA ARGUMENTACIÓN}

Linaloe León Baro doi: https://doi.org/10.36799/el.v5i1.102 Volumen 5, Número 1, Año 2020, ISSN: 2448-5942 
La argumentación es una actividad discursiva que se presenta constantemente en la vida cotidiana; argumentamos en entrevistas de empleo, en debates, congresos, salones de clase, etcétera. Por lo tanto, podría decirse que los debates son el eje de la vida social, ya que las diferencias de opinión surgen constantemente en todas las áreas de la vida pública, ya sea con respecto a tópicos importantes o hechos triviales.

Es un tipo de discurso que conlleva un proceso de razonamiento en el que alguien defiende una opinión personal sobre un tema particular, de forma oral o escrita, con la intención de convencer a un público de aceptar o adoptar su punto de vista. Para esto, debe ofrecer razones que evidencien la aceptabilidad de su perspectiva, además de adelantar la posición de su audiencia y considerar visiones opuestas para poder refutar los posibles contraargumentos, por lo que necesita además manejar con eficiencia los recursos lingüísticos que sirvan a la expresión de la argumentación (Calsamiglia y Tusón, 2004; Felton y Herko, 2004; Rodríguez, 2004; Van Eemeren et al, 1997, 2004, 2006).

\section{EL MODELO DE TOULMIN}

El Modelo de Toulmin ha sido muy utilizado como base para la realización de investigaciones sobre argumentación oral y escrita, ya que fue diseñado con la intención de que fuera útil para el análisis de este tipo de discurso en cualquier área o disciplina. El modelo surgió de las observaciones del autor sobre la racionalidad de algunos procedimientos legales, aunque afirmaba que la discusión argumentativa procedía de igual forma en todos los campos de la argumentación y, por lo tanto, podía aplicarse el modelo en cualquier tipo de disciplina abierta al debate (Toulmin, 2003; Rodríguez Bello, 2004; Van Eemeren y Grootendorst, 2004).

En su propuesta, Toulmin deja de lado la idea de la "validez formal" de la lógica, pues la considera un criterio únicamente aplicable a los argumentos analíticos, que son raros en el uso real (Van Eemeren y Grootendorst, 2004). A partir de sus observaciones y razonamientos en el campo de lo legal, señala que para comprender la naturaleza y validez de un argumento es necesario considerar una mayor diversidad de distinciones, por lo que sería necesario emplear un esquema y patrón de análisis que represente los argumentos de manera más elaborada, no sólo en lo legal, sino en cualquier área abierta a la discusión, véase el cuadro 1.

Linaloe León Baro doi: https://doi.org/10.36799/el.v5i1.102 Volumen 5, Número 1, Año 2020, ISSN: 2448-5942 
Los elementos que Toulmin propone como parte de un argumento son:

\begin{tabular}{|l|l|}
\hline Afirmación (Claim): & La idea que se va a defender. \\
\hline Datos (Data): & Evidencia que respalda la afirmación. \\
\hline Garantías (Warrant): & $\begin{array}{l}\text { Enunciados de carácter general que } \\
\text { sirven como puente entre la afirmación y } \\
\text { los datos. }\end{array}$ \\
\hline Respaldo (Backing): & $\begin{array}{l}\text { Ideas adicionales que sirven para precisar } \\
\text { y apoyar las garantías. }\end{array}$ \\
\hline $\begin{array}{l}\text { Matizadores modales } \\
\text { (Modal qualifiers): }\end{array}$ & Indicadores de la fuerza de las garantías. \\
\hline $\begin{array}{l}\text { Condiciones de excepción } \\
\text { o de refutación } \\
\text { (Reservation/Rebuttals): }\end{array}$ & $\begin{array}{l}\text { Las circunstancias en las que ha de dejarse } \\
\text { de lado la autoridad de las garantías. }\end{array}$ \\
\hline
\end{tabular}

Cuadro 1. Componentes de un argumento en el modelo de Toulmin

\section{EL MARCO PRAGMA-DIALÉCTICO DE LA ARGUMENTACIÓN}

El Marco pragma-dialéctico de Van Eemeren, et al $(2004,2006)$ asume que el uso argumentativo de la lengua es, en principio, un intercambio de puntos de vista entre dos partes con distintas opiniones, aun cuando se dé en forma de monólogo, tomado éste como un tipo específico de discusión crítica en el que el protagonista habla o escribe y el rol del antagonista permanece implícito.

En la argumentación entonces hay un punto de vista sostenido por el hablante o el escritor, quien lo defiende por medio de la argumentación ante un oyente o lector que duda de su aceptabilidad o piensa distinto. Entonces, los autores definen la argumentación como una actividad verbal, social y racional encaminada a convencer a una audiencia racional y crítica de que un punto de vista es aceptable, a partir de la exposición de una constelación de proposiciones que justifican o refutan la proposición que expresa el punto de vista (Van Eemeren y Grootendorst, 2004). Las expresiones que conforman esa constelación constituyen un acto de habla complejo orientado a convencer a un crítico racional, por lo tanto, la argumentación es un llamado a la racionalidad, ya que se asume que el oyente o el lector actuarán críticamente cuando al evaluar los argumentos presentes.

En esta teoría de la argumentación, consideran que los desacuerdos entre las personas propician constantemente la argumentación, ya que difícilmente se aceptan las diferencias de opinión sin intentar llegar a un acuerdo razonable. Así, cuando se utiliza la argumentación como 
un medio para resolver esa diferencia, mantienen una discusión argumentativa, en las que los participantes deberán exponer sus puntos de vista y las bases que lo sustentan.

Cuando se intenta determinar la calidad de una discusión de este tipo, es necesario que se tomen en cuenta todos los elementos relevantes que participen en el desarrollo de la discusión y analizar su pertinencia y razonabilidad. Esta tarea podría complicarse en los casos en los que la argumentación se presenta en forma de monólogo, como es el caso de la forma escrita, ya que es difícil reconocer los elementos de la discusión; sin embargo, aun en esta forma es posible reconocer el punto de vista y los argumentos que se utilizan para convencer a los críticos potenciales, esto a través de la estructura y contenido general del texto y los elementos lingüísticos que sirven a la argumentación.

El marco pragma-dialéctico de la argumentación sostiene que los argumentos pueden presentar diversos tipos de estructuras. Una de ellas es la argumentación simple, que se presenta cuando se ofrece sólo una razón a favor o en contra de un punto de vista, pero también pueden presentarse estructuras más complejas al añadir un mayor número de razones, que pueden relacionarse entre sí de diversas maneras, y además dependiendo de cómo se organiza la defensa de una postura al anticipar las dudas o críticas de la oposición.

En la argumentación con estructura más compleja, se ofrecen varias razones para defender un punto de vista y las relaciones que presentan entre ellas determinan el tipo de estructura del argumento. La argumentación múltiple es aquella en la que las razones pueden considerarse defensas alternativas independientes entre sí, ya que cada una podría sostenerse sola teóricamente y considerarse suficiente para sostener la postura. Por otra parte, en la argumentación coordinada las razones pueden interpretarse como interdependientes, de modo que se forme una "cadena paralela" de razones que se refuerzan mutuamente, ya sea porque el argumento previo es débil y, por lo tanto, insuficiente para sustentar solo el punto de vista o para prevenir posibles objeciones sobre el primer argumento dado. Por último, como tercer tipo de estructura compleja está la argumentación subordinada, donde se forma una "cadena serial" de razones que se apoyan unas a otras, esto ocurre cuando un argumento no es suficiente para sostener un punto de vista, por lo que necesita otro en el cual apoyarse para que la defensa sea concluyente.

\section{METODOLOGÍA}

Linaloe León Baro doi: https://doi.org/10.36799/el.v5i1.102 Volumen 5, Número 1, Año 2020, ISSN: 2448-5942 
En el presente documento, se reportan avances de investigación en su fase descriptiva, con el propósito de conocer más sobre el manejo del discurso argumentativo escrito por parte de estudiantes universitarios; esto a través de un análisis de los elementos que incluyen en la construcción de los argumentos con los que defienden su punto de vista sobre un tema particular. Para esto, se identificaron y cuantificaron los elementos a los que recurrieron en cada una de las versiones de sus textos, lo que además permite observar los cambios estructurales entre los dos escritos de cada estudiante.

\section{PARTICIPANTES Y CORPUS}

Durante la etapa de recolección de datos para el trabajo de investigación original, se contó con la colaboración de un total de 58 estudiantes de primer año de dos carreras de la Universidad de Sonora; 24 alumnos de la Licenciatura en Lingüística y 34 de la Licenciatura en la Enseñanza del Inglés. En este documento, se reportarán únicamente los avances con respecto al análisis de los textos producidos por los estudiantes de Lingüística. El contacto con los estudiantes se estableció por medio de uno de sus profesores de asignatura, quien autorizó el acceso al grupo durante una hora de su curso; para la segunda sesión, se acordó con los estudiantes trabajar en una hora libre dentro de su carga horaria en la misma semana.

A partir de las dos sesiones de recopilación de datos con el grupo de esta carrera, se obtuvo un total de 34 escritos, sin embargo, para efectos de la investigación se seleccionaron solamente los textos de los estudiantes que sí se presentaron a las dos reuniones de trabajo y de aquellos que hayan seguido adecuadamente las instrucciones en ambas sesiones. Por lo tanto, el corpus total analizado en este reporte está conformado por 28 textos en total, resultado de la suma de las dos versiones de los textos escritos por 14 estudiantes.

\section{ACTIVIDAD DE ESCRITURA}

Durante el diseño de la metodología utilizada para esta fase de la investigación, el primer paso fue definir el tema sobre el que los estudiantes tendrían que argumentar en sus textos; después de analizar las posibles opciones, el tema elegido fue "el impacto de las redes sociales", ya que se consideró como una problemática al alcance de todos los estudiantes, en tanto que están en Linaloe León Baro doi: https://doi.org/10.36799/el.v5i1.102 Volumen 5, Número 1, Año 2020, ISSN: 2448-5942 
constante exposición a las redes sociales independientemente de que sean o no usuarios frecuentes de estos medios, por lo que podrían abordar el análisis crítico del tema con relativa facilidad.

Las actividades estuvieron divididas en dos sesiones. En la primera, los estudiantes realizaron la lectura de un fragmento del artículo "Redes sociales y estudiantes universitarios: del nativo digital al informívoro saludable" ${ }^{2}$, con el fin de que conocieran la polémica que tratarían. Posteriormente, recibieron una hoja de actividad con el siguiente contenido:

\begin{abstract}
ACTIVIDAD
Las redes sociales en línea son una de las herramientas tecnológicas más utilizadas actualmente por los estudiantes de nivel medio superior y superior; asimismo, algunas empresas empiezan a hacer uso de ellas para difundir y manejar sus negocios. Una parte de la sociedad tiene la impresión de que son sólo una pérdida de tiempo, un ejemplo de esto son los padres de familia que limitan o condicionan el tiempo que dedican sus hijos a las redes; pero, por otro lado, algunas investigaciones demuestran que bien utilizadas pueden traer grandes beneficios.

Desde tu punto de vista como joven estudiante... ¿Consideras que el creciente uso de las redes sociales impacta positiva o negativamente en la sociedad? Expresa y defiende tu opinión al respecto.
\end{abstract}

La instrucción fue leída en voz alta y se resolvieron en grupo las dudas de los estudiantes antes de que comenzaran a redactar; la elaboración de los textos tomó alrededor de 30 minutos.

La segunda sesión, realizada dos días después, inició con una breve plática grupal acerca de las redes sociales para asegurar que todos tuvieran claridad sobre el tema, ya que al revisar los primeros textos se observó que algunos alumnos tenían confusión sobre la delimitación de estos medios. Posteriormente, se realizó una discusión oral sobre los puntos a favor y en contra de las redes sociales con respecto al impacto que tienen en la sociedad. La discusión tuvo formato de debate, donde los estudiantes se dividieron en dos grupos, a cada uno de los cuales se le asignó al azar la postura que defendería, independientemente del punto de vista de cada integrante. La actividad tuvo una duración aproximada de 25 minutos, tiempo en el que los estudiantes se mostraron muy activos al momento de expresar sus puntos de vista y responder a las ideas planteadas por sus demás compañeros.

Al finalizar la discusión, se les presentó una breve exposición de 10 minutos sobre el discurso argumentativo, haciendo mención de sus características y los componentes que pueden

\footnotetext{
${ }^{2}$ Valerio Ureña, G. y Valenzuela Gonzáles, R. (2011). http://eprints.rclis.org/16267/1/Informivoro.pdf Linaloe León Baro doi: https://doi.org/10.36799/el.v5i1.102 Volumen 5, Número 1, Año 2020, ISSN: 2448-5942
} 
ser utilizados para estructurar los argumentos; al mismo tiempo, se iba relacionando esa información con la experiencia que tuvieron al argumentar de forma oral. Por último, los estudiantes elaboraron una segunda versión de sus textos, tomando en cuenta lo que advirtieron durante el debate oral y la exposición, basándose además en el primer texto que escribieron.

\section{PROCEDIMIENTO DE ANÁLISIS}

El procedimiento utilizado para el análisis de la estructura argumentativo de los textos se basó en la propuesta de graficación de Ferretti, Lewis y Andrews-Weckerly (2009), utilizada después por Chase (2011), y que se deriva, a modo de adaptación, del marco pragma-dialéctico de la argumentación de Van Eemeren, et al (2004, 2006). Este método de esquematización fue seleccionado debido a que permite apreciar con mayor claridad la estructura de los textos al hacer visibles los distintos elementos argumentativos que los conforman y la manera en la que éstos se relacionan entre sí para formar la defensa del punto de vista expresado por el escritor.

En la propuesta se distingue entre elementos funcionales y no funcionales. Dentro de los funcionales se encuentran elementos que corresponden particularmente al razonamiento argumentativo:

- Punto de vista: la postura del escritor sobre el tema que se plantea.

- Raz̧ones: información que utiliza el escritor para sustentar la defensa de su punto de vista. Es preciso distinguir entre razones de nivel 1, que son las que apoyan directamente el punto de vista, y razones subordinadas, que cumplen la función de respaldar razones previas. También es necesario identificar aquellas que funcionan como sustento de algún otro tipo de elemento argumentativo.

- Puntos de vista alternativos: presentación de la postura opuesta a la del punto de vista del escritor.

- Contraargumentos: crítica u objeción que puede utilizarse para debilitar el punto de vista de alguien. En la argumentación escrita pueden considerarse críticas potenciales del punto de vista del escritor y como un soporte para un punto de vista alternativo.

Linaloe León Baro doi: https://doi.org/10.36799/el.v5i1.102 Volumen 5, Número 1, Año 2020, ISSN: 2448-5942 
- Refutación: proposición que refuta o debilita un punto de vista alternativo o contraargumento con el fin de fortalecer la postura del escritor.

Además, dentro de los elementos funcionales se toman en cuenta otros elementos propios de la construcción textual que contribuyen a darle mayor forma a la composición:

- Marcadores funcionales: elementos que sirven a un propósito particular para el escritor, a menudo se usa como una transición para introducir razones, argumentos y puntos de vista.

- Repeticiones retóricamente funcionales: ocurren cuando el escritor reafirma razones o puntos de vista expresados anteriormente.

- Introducción: se define como un anuncio de lo que seguirá en la presentación del argumento del escritor. Puede ser sólo una oración o varias.

- Conclusión: está presente cuando el escritor hace un cierre o resumen sobre lo que ha escrito a lo largo de su ensayo.

Por último, los elementos no funcionales son aquellas unidades de texto que no juegan ningún rol en la defensa del punto de vista del escritor, casos de este tipo de elementos serían: (a) las repeticiones que no cumplen una función en el texto y (b) la información que no es relevante para el tema. También entran en esta categoría los fragmentos ilegibles del texto si es escrito a mano o información que no tiene sentido claro.

Además de considerar estos elementos para la esquematización, también se consideraron los diversos tipos de estructuras que pueden surgir a partir del modo en el que se relacionan los componentes dentro del texto. Van Eemeren et al. (2006), mencionan que la argumentación puede presentar diversas estructuras. Puede ser simple, cuando hay sólo una razón explícita a favor o en contra de un punto de vista, pero también pueden presentarse estructuras complejas dependiendo de cómo se organiza la defensa de una postura al anticipar las dudas o críticas de la oposición.

En la argumentación con estructura compleja, se ofrecen varias razones para apoyar o rechazar un punto de vista, Van Eemeren, et al (2006) proponen la existencia de tres tipos de estructuras de argumentación complejas: a) argumentación múltiple: las razones pueden ser defensas alternativas independientes, b) argumentación coordinada: las razones pueden interpretarse como Linaloe León Baro doi: https://doi.org/10.36799/el.v5i1.102 Volumen 5, Número 1, Año 2020, ISSN: 2448-5942 
interdependientes, de modo que se forme una "cadena paralela" de razones mutuamente reforzadas y c) argumentación subordinada: se forma una "cadena serial" de razones que se apoyan unas a otras.

\section{RESULTADOS}

Con el fin de conocer la forma en la que los estudiantes estructuran sus textos argumentativos, se esquematizó la totalidad de los ensayos escritos por los estudiantes que participaron en las dos sesiones de trabajo que se realizaron para la recolección de datos. Los 28 ensayos fueron graficados siguiendo el procedimiento descrito anteriormente, lo que permitió observar con claridad los distintos elementos argumentativos que utilizaron para construir la defensa de sus puntos de vista.

A continuación, se presenta un ejemplo de uno de los textos del corpus, el cual fue resultado de la segunda sesión de trabajo; se incluye además su respectivo esquema (Figura 1) y la descripción del mismo.

(1) [I. El hablar de una temática tan variada en opiniones como las redes sociales y sus usos, tendríamos que mantener la cabeza fría para elegir la postura], [PV1. en mi caso estoy a favor], [PV1.R1a porque puedo hablar con mi familia] [PV1.R1b y verlos] [CA1 tal vez el no sentir el contacto físico no llene "un vacío"] [CA1.REF1 pero esto nos denota el principal uso para el cual fueron creadas, que es romper barreras,] [CA1.REF2 acabar con las distancias] [CA1.REF3 y mejorar la comunicación en los habitantes del planeta,] [PV1.R2a el simple hecho de que sea posible ver a personas que están al otro lado del mundo,] [PV1.R2b o que nos enteremos de lo que está pasando a miles de kilómetros de distancia de donde nos encontramos es fascinante], [MF el punto es:] [PV2 no podemos culpar a las redes sociales del mal uso que se les da] [PV2.R1 ya que es la misma sociedad la que les da el uso para fines correctos o incorrectos.]

[PV2.R1.R1 Nosotros como usuarios de las redes sociales tendríamos que tener una mejor educación], [CA2. y sí tal vez nuestros padres o personas de alrededor no nos la den,] [CA2.REF pero ahí mismo en las redes existe una infinidad de información para aprender el uso correcto de ellas.]

Linaloe León Baro doi: https://doi.org/10.36799/el.v5i1.102 Volumen 5, Número 1, Año 2020, ISSN: 2448-5942 
[C. Si en algún momento se culpa a las redes sociales por las consecuencias malas de su uso es sólo un indicador de cómo va la sociedad siempre busca culpables para sus actos en lugar de aceptar la responsabilidad.]

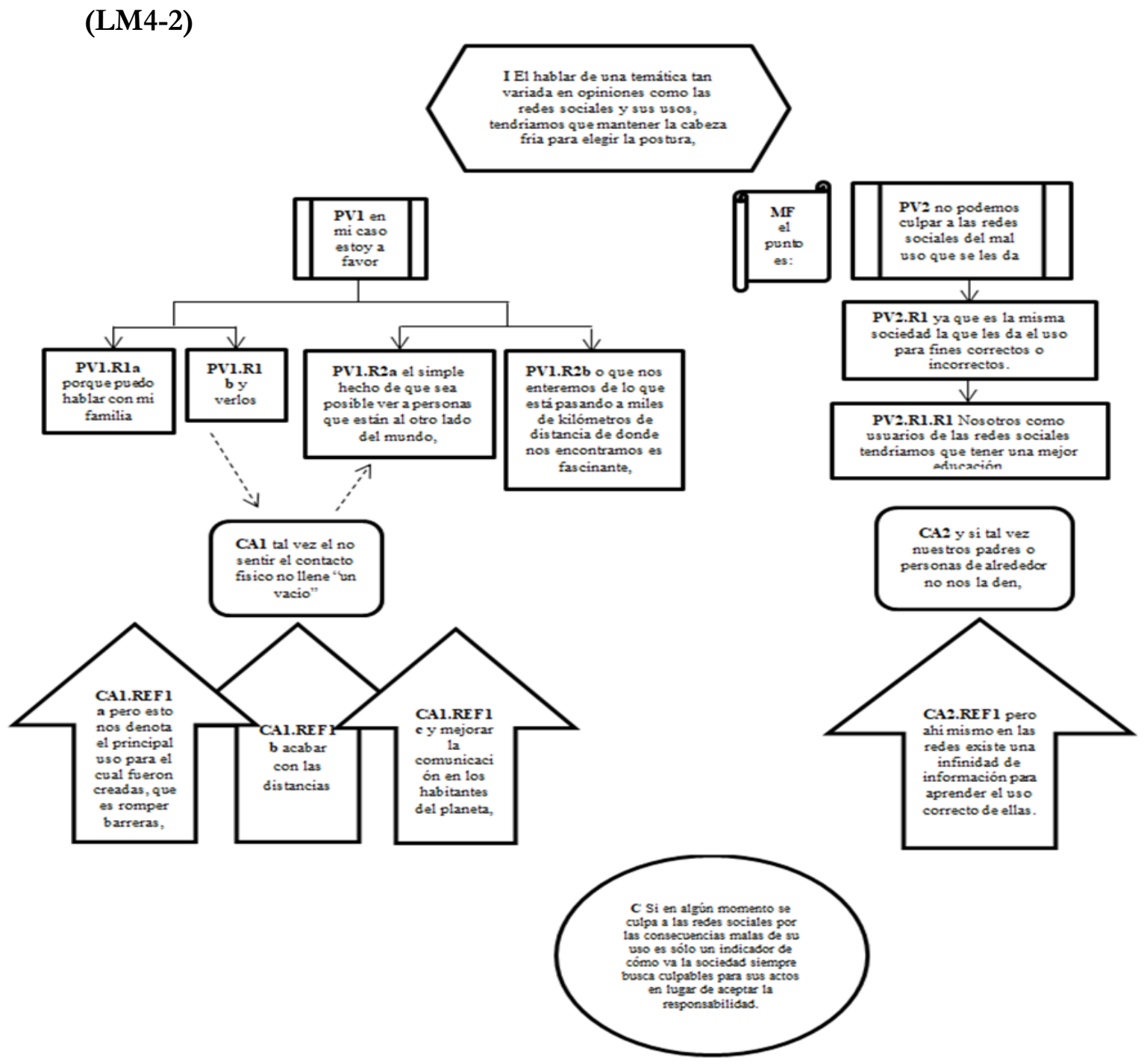

Figura 1. Esquematización del texto escrito por el estudiante LM4 en la segunda sesión de trabajo Abreviaturas: I=Introducción, PV1=Punto de vista 1, PV1.R1a/PV1.R1b=Primera cadena coordinada de razones de nivel 1, PV1.R2a/PV1.R2b=Segunda cadena coordinada de razones de primer nivel, $\mathrm{CA} 1=$ Contraargumento 1, CA1.REF1a/REF1b/REF1c $=$ Cadena coordinada de refutaciones, PV2=Punto de vista 2, PV2.R1/PV2.R1.R1=Cadena coordinada de razones, CA2=Contraargumento 2, CA2.REF1=Refutación para contraargumento $2, \mathrm{C}=$ Conclusión

En el texto presentado en (1) la alumna comienza con una breve introducción para después presentar su punto de vista, en el que explícitamente menciona estar a favor con respecto Linaloe León Baro doi: https://doi.org/10.36799/el.v5i1.102 Volumen 5, Número 1, Año 2020, ISSN: 2448-5942 
al uso de las redes sociales. Posteriormente, presenta dos razones de primer nivel relacionadas entre sí, por lo que forman una cadena de razones coordinadas; después está presente el contraargumento < tal vez el no sentir contacto físico no llene "un vacío">, que se considera como la anticipación de una posible crítica de la audiencia y que podría restar fuerza a su punto de vista personal, pero esto se evita gracias a la cadena de tres refutaciones que ofrece después la estudiante para debilitar el contraargumento. El texto continúa con la adición de dos razones más de primer nivel que también forman una cadena coordinada, seguidos por un marcador funcional de transición que introduce una proposición con carga personal, por lo que es graficado como un punto de vista y que, además, está sustentado por una razón de primer nivel y una más que se subordina a ésta; en este punto hace mención de un contraargumento más, el cual también es refutado. Por último, presenta una conclusión que sirve como cierre del texto y al mismo tiempo refuerza los argumentos que presentó para defender su postura personal ante el tema puesto a discusión.

A continuación, se presentan los resultados que se han obtenido hasta este momento a partir de la esquematización y análisis de los 28 textos que conforman el corpus seleccionado para este reporte de investigación. Primeramente, se ofrece una exposición cuantitativa y descriptiva general sobre los distintos tipos de elementos que utilizaron los estudiantes en las distintas versiones de sus textos y la frecuencia de uso de dichos elementos, para después describir brevemente el uso de los diferentes componentes en particular; asimismo, serán señaladas algunas de las diferencias observadas entre los textos de la primera y la segunda sesión de escritura.

$\mathrm{Al}$ identificar y cuantificar los distintos elementos que los estudiantes incluyeron en sus textos, se registró un total de 8 elementos funcionales distintos: punto de vista, razones, puntos de vista alternativos, contraargumentos, refutaciones, introducción, conclusión y marcador funcional. No fue identificado entre los textos ninguna unidad no funcional. La siguiente gráfica 1 concentra los resultados obtenidos de esta cuantificación de los elementos identificados dentro de los textos de primera versión, representados en las columnas izquierdas, y los de segunda versión, que se presentan en las columnas del lado derecho.

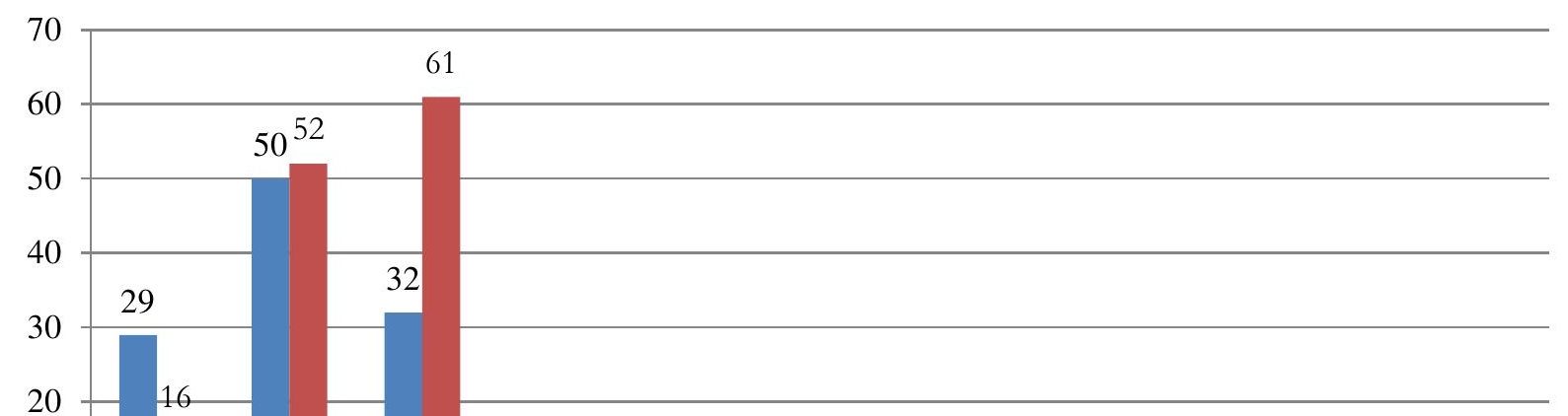


Gráfica 1. Elementos funcionales en 1ra. y 2da. versión de los textos

En los 14 textos resultantes de la primera sesión de escritura, dentro de los elementos del razonamiento argumentativo se registraron 29 unidades de texto en función de puntos de vista y 82 razones, de las que 50 corresponden a razones de primer nivel y 32 a razones subordinadas. Además, se registró 1 punto de vista alternativo y 2 refutaciones. En el caso de los elementos de construcción textual considerados dentro de los elementos funcionales, se encontró que fueron 3 estudiantes los que abrieron su texto con una introducción, 8 cerraron con una conclusión y además se registraron 4 marcadores funcionales.

En la tabla 1 puede observarse que en 9 de los 14 textos se registraron varios puntos de vista, esto no quiere decir que los alumnos tengan tres o cuatro opiniones completamente distintas sobre el tema, sino que mostraron una postura tanto a favor como en contra y, para efectos de la graficación, fue necesario representar por separado las distintas unidades de texto que estaban involucradas en la expresión global del punto de vista. En cuanto a las razones, el texto con mayor número de razones de nivel 1 incluyó 9, mientras que los que contaron con menos de ellas contenían 2; además, en 10 de los 14 textos se encontraron razones subordinadas, que sirven como complemento y respaldo de las de nivel 1, el texto con mayor número de éstas Linaloe León Baro doi: https://doi.org/10.36799/el.v5i1.102 Volumen 5, Número 1, Año 2020, ISSN: 2448-5942 
contó con 7 y los que menos las utilizaron incluyeron una. Los elementos como puntos de vista argumentativos y refutaciones fueron encontrados únicamente en un texto del corpus, presentándose una y dos veces respectivamente; en ningún escrito de primera versión se registraron contraargumentos.

En la siguiente tabla (tabla 1) se muestra el conteo total de elementos argumentativos y textuales utilizados por cada uno de los estudiantes en la primera versión de sus textos:

\begin{tabular}{|c|c|c|c|c|c|c|c|c|}
\hline $\begin{array}{l}\text { Clave de } \\
\text { estudiante }\end{array}$ & $\begin{array}{l}\pi \\
0 \\
0 \\
5 \\
0 \\
0 \\
0 \\
0 \\
0 \\
0 \\
0 \\
0\end{array}$ & 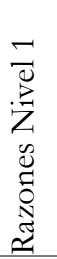 & 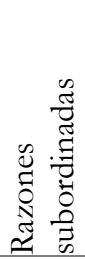 & 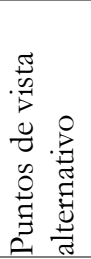 & 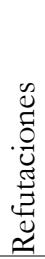 & 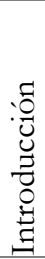 & 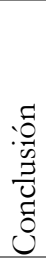 & 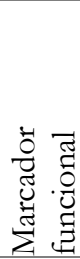 \\
\hline LH1 & 3 & 5 & 2 & - & - & - & 1 & 1 \\
\hline LH2 & 3 & 5 & 3 & - & - & - & 1 & - \\
\hline LH3 & 3 & 9 & - & - & - & - & - & - \\
\hline LH4 & 2 & 3 & 1 & - & - & - & 1 & - \\
\hline LH5 & 1 & 2 & - & - & - & - & - & - \\
\hline LM1 & 3 & 5 & 5 & - & - & - & 1 & - \\
\hline LM2 & 4 & 4 & 7 & - & - & - & 1 & 1 \\
\hline LM3 & 1 & 4 & 3 & - & - & - & - & - \\
\hline LM4 & 1 & 2 & 4 & - & - & - & - & 1 \\
\hline LM5 & 2 & 3 & 1 & - & - & 1 & 1 & - \\
\hline LM6 & 2 & 2 & 3 & - & - & 1 & 1 & - \\
\hline LM7 & 1 & 2 & - & 1 & 2 & - & - & - \\
\hline LM8 & 2 & 4 & 3 & - & - & - & 1 & - \\
\hline LM9 & 1 & - & - & - & - & 1 & - & - \\
\hline Totales & 29 & 50 & 32 & 1 & 2 & 3 & 8 & 3 \\
\hline
\end{tabular}

Tabla 1. Elementos argumentativos en textos de primera versión

Por otra parte, en el caso de los textos obtenidos durante la segunda sesión de trabajo, la cantidad de fragmentos textuales donde se reflejaba el punto de vista de los estudiantes se redujo a 16. Con respecto a las razones, la cifra total aumentó a 124, 52 de éstas corresponden a razones de nivel 1 directamente ligadas al punto de vista y 61 fueron razones subordinadas a éstas; se encontraron además 6 razones de nivel 1 que estaban ligadas a otro tipo de elementos argumentativos distintos de los puntos de vista y 5 subordinadas a éstas. En cuanto a los demás componentes de la argumentación, puede observarse un aumento general, pues de 1 punto de 
vista argumentativo registrado en las primeras versiones, se pasó a 4 usos de este elemento en la segunda versión, se identificaron también 3 contraargumentos, que en las primeras versiones no estuvieron presentes $\mathrm{y}$, por su parte, las refutaciones aumentaron su cantidad total de usos de 2 a 11. Por último, los resultados con respecto a los elementos de carácter textual, se encontró que 3 estudiantes abrieron su texto con una introducción, 6 cerraron con una conclusión y además fueron identificados 4 marcadores funcionales.

\begin{tabular}{|c|c|c|c|c|c|c|c|c|c|c|c|}
\hline $\begin{array}{l}\text { Clave de } \\
\text { estudiante }\end{array}$ & $\begin{array}{l}\frac{\pi}{5} \\
.0 \\
5 \\
0 \\
0 \\
0 \\
0 \\
0 \\
\Xi \\
\Xi \\
0\end{array}$ & 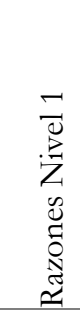 & 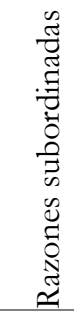 & 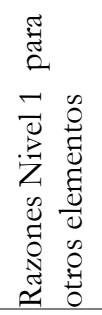 & 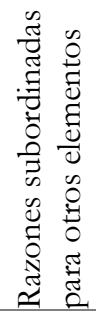 & 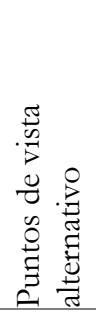 & 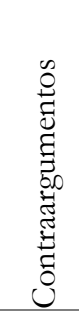 & 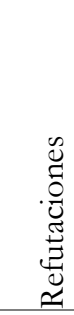 & 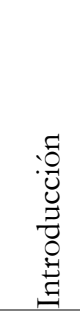 & 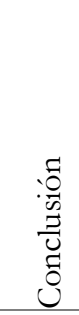 & 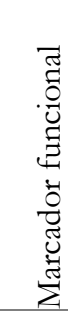 \\
\hline LH1 & 1 & 4 & 6 & 2 & - & - & 1 & 1 & - & 1 & - \\
\hline LH2 & 1 & 1 & 5 & - & - & - & - & - & - & 1 & - \\
\hline LH3 & 1 & 3 & 6 & - & - & - & - & - & - & - & - \\
\hline LH4 & 1 & 2 & 4 & - & - & - & - & - & - & - & 1 \\
\hline LH5 & 1 & - & - & 2 & - & 1 & - & 1 & - & - & - \\
\hline LM1 & 1 & 5 & 9 & - & - & - & - & - & - & 1 & - \\
\hline LM2 & 2 & 7 & 10 & - & - & - & - & - & - & - & - \\
\hline LM3 & 1 & 2 & 5 & - & - & - & - & - & - & - & - \\
\hline LM4 & 2 & 5 & 1 & - & - & - & 2 & 4 & 1 & 1 & 1 \\
\hline LM5 & 1 & 5 & 4 & - & - & 1 & - & 2 & - & - & - \\
\hline LM6 & 1 & 7 & & 2 & 5 & 1 & - & 1 & - & 1 & 2 \\
\hline LM7 & 1 & 3 & 6 & - & - & - & - & - & - & - & - \\
\hline LM8 & 1 & 3 & 5 & - & - & - & - & - & 1 & 1 & - \\
\hline LM9 & 1 & 5 & - & - & - & 1 & - & 2 & 1 & - & - \\
\hline Totales & 16 & 52 & 61 & 6 & 5 & 4 & 3 & 11 & 3 & 6 & 4 \\
\hline
\end{tabular}

Tabla 2. Elementos argumentativos en textos de segunda versión

En la tabla 2 puede apreciarse la contabilización de los elementos funcionales argumentativos y de orden textual que fueron utilizados por cada uno de los estudiantes en la segunda versión de sus textos. En la columna destinada a los puntos de vista, puede observarse con claridad que en la mayoría de los escritos se identificó una única unidad de texto con la función de expresar el punto de vista, esto se debe a que ya no manifestaron tener una opinión tanto en contra como a favor, sino que asumieron una postura unilateral ante el tema. Es posible observar también el Linaloe León Baro doi: https://doi.org/10.36799/el.v5i1.102 Volumen 5, Número 1, Año 2020, ISSN: 2448-5942 
aumento general en la cantidad de razones de nivel 1 y las subordinadas a éstas, pero la mayor diferencia es la aparición de las razones que estuvieron sirviendo como respaldo para otro tipo de elementos argumentativos distintos de los puntos de vista, tal es el caso de las refutaciones, y que éstas incluso llegaron a estar respaldadas por razones subordinadas.

Otra diferencia observada es la presencia de los contraargumentos, elementos que no estuvieron presentes entre los textos elaborados en la primera sesión de escritura; fueron dos los estudiantes que incluyeron este tipo de componente argumentativo en la segunda versión de sus textos. También hubo un aumento en la inclusión de puntos de vista alternativos, que consisten en la expresión de la postura directamente contraria a la personal, pues en la primera versión fueron utilizados sólo por una persona y en la segunda, por cuatro. Las refutaciones por su parte incrementaron su uso al presentarse esta vez en 11 ocasiones, esto podría derivarse del aumento de los dos componentes anteriormente mencionados, pues las refutaciones son proposiciones utilizadas para debilitar la fuerza de los puntos de vista alternativos y los contraargumentos con el fin de fortalecer la postura del escritor. En cuanto a los elementos funcionales de carácter textual no se registró una mayor variación en su frecuencia de uso entre la primera y segunda versión de los escritos.

Además de la identificación y contabilización general de los datos, se estudió el desempeño de los estudiantes a partir de un análisis no sólo sobre la cantidad de componentes que utilizaron en ambos textos, sino también considerando la diversidad de elementos funcionales que incluyeron. Las columnas izquierdas de la gráfica 2 están referidas a la cantidad total de elementos utilizados, en ellas es posible observar que 11 de los 14 estudiantes mostraron un avance al haber incluido una mayor cantidad total de elementos en sus textos de segunda versión, mientras que 1 se mantuvo en la misma cantidad y 2 mostraron un retroceso al disminuir la cantidad de componentes. Por otro lado, en las columnas de la derecha se representa el desempeño de los estudiantes con respecto a la diversidad de componentes funcionales que utilizaron, en ellas se muestra que 7 estudiantes avanzaron al incluir una mayor diversidad de elementos en la segunda versión de sus textos, 5 no presentaron cambios en la cantidad de componentes distintos y 3 retrocedieron al haber incluido una menor diversidad de elementos en su segunda producción textual.

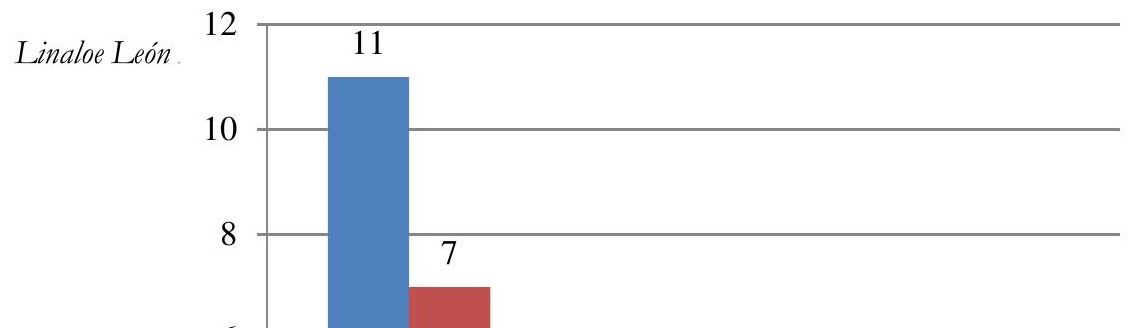




\section{Gráfica 2. Desempeño}

Finalmente, para concluir este apartado de resultados, se presentan como ejemplo los textos producidos por una de las estudiantes del grupo, con el fin de que los esquemas (Figura 2) ofrezcan una visión más clara sobre el procedimiento de graficación y cómo esto facilita el análisis de la estructura general de los textos y los cambios que se presentan:

(2) [I En diferentes puntos de vista, todo depende de la manera en la que se utilizan las redes, todo en exceso es malo.] [PV1 Pienso que a nadie le hace daño tener facebook, Twitter, etc.] [R1 en cierto modo es una manera de relajación,] [R1.R1 al menos para mi si lo es.] [PV2 Claro que si todo mi tiempo fuese dedicado al internet ese y sería un problema], [R1 ya que afectaría mi vida], [R1.R1a como el dejar de hacer otro tipo de actividades que son más importantes], [R1.R1b además de que el riesgo de convertirme en adicta a las redes también sería un gran problema]. [C Es por eso que digo que todo en equilibrio es mejor.] (LM6-1)

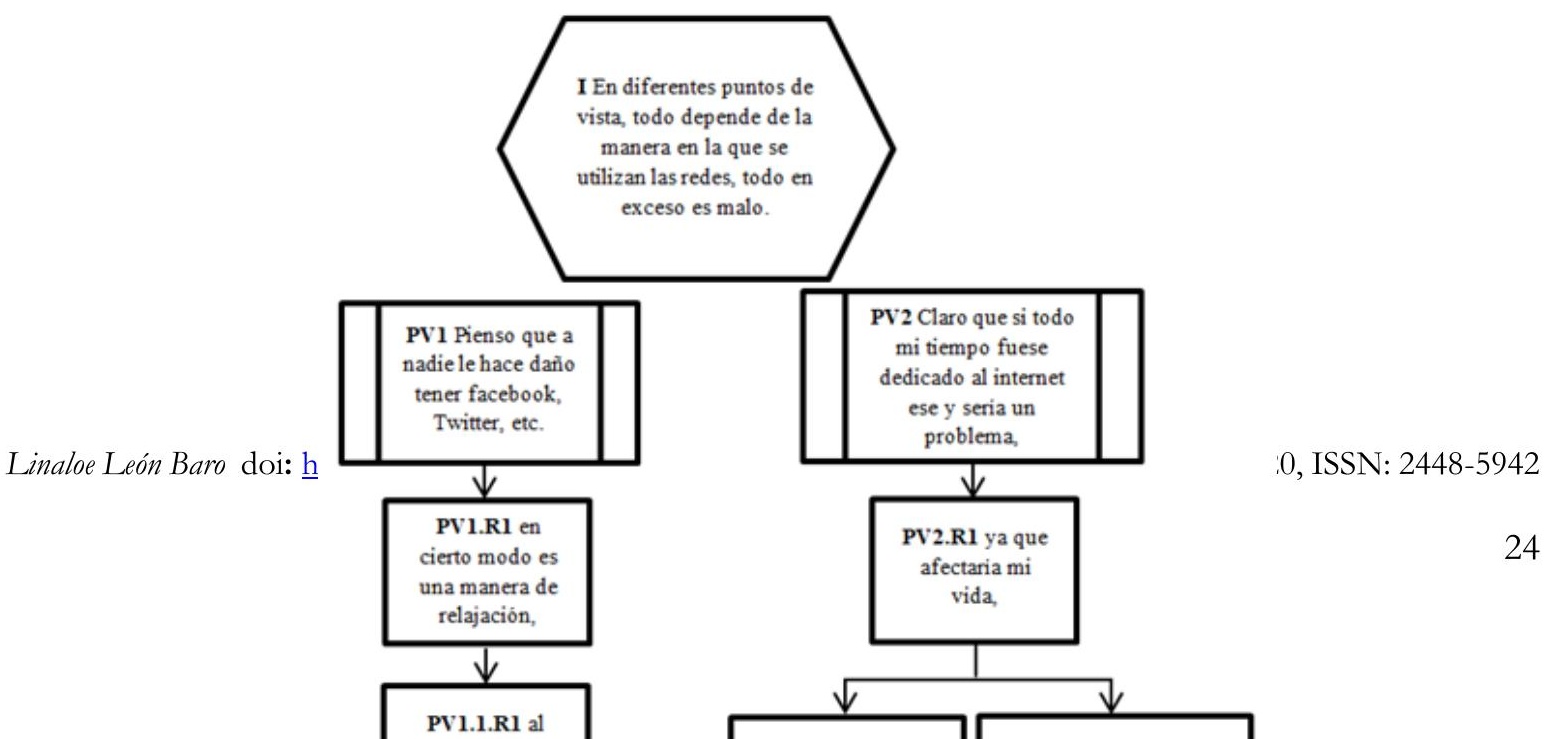


Figura 2. Esquema del texto producido por estudiante LM6 en la primera sesión de escritura

(3) [PV1 El lado o el punto de vista que yo en mi manera de ver la situación es la correcta sería la positiva.]

[PVA Sé que hay mucha polémica al respecto sobre las personas que hacen mal uso de las redes sociales,][REF pero si nos fijamos o adentramos más, en realidad las personas son las mismas causantes de ello], [REF.R1 "uno cosecha lo que siembra",] [MF A qué voy con esto?] [REF.R1.R1 Bueno, si publico una imágen semidesnuda] [MF qué consigo?] [REF.R1.R1.R1a Críticas,] [REF.R1.R1.R1b malos comentarios, [REF.R1.R1.R1c incluso mi vida correría peligro.]

[REF.R2 Si eres padre o madre de familia debes saber y estar al tanto de la situación que tus hijos viven fuera de casa y en internet,] [REF. R2.R1 así se evitarían muchos conflictos.]

[PV1.R1 Las redes han influido para encontrar a personas desaparecidas,] [PV1.R2 compra-venta de cosas,] [PV1.R3a comunicarnos con nuestros amigos], [PV1.R3b familiares lejanos], [PV1.R4 publicidad gratis], [PV1.R5 poder expresarnos libremente], [PV1.R6 compartir nuestros momentos importantes de los demás], [C esto entre muchas cosas más es por lo que es para mí positivo.]

Lina

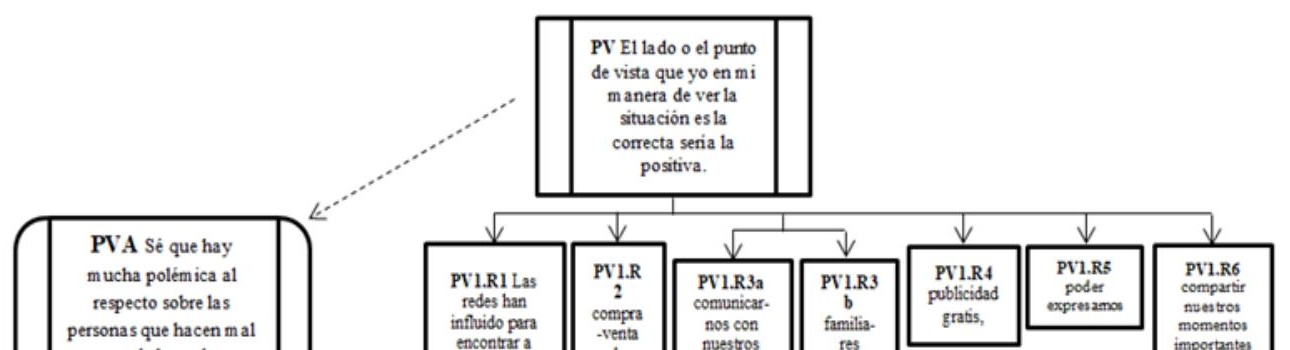


Figura 3. Esquema del texto producido por estudiante LM6 en la segunda sesión de escritura

En el ejemplo número (2) (figura 2) se presenta el texto que la alumna escribió en la primera sesión de escritura, en el que incluyó un total de 9 componentes y una diversidad de 5 . En este texto, la estudiante inició con una breve introducción, después mostró una postura dividida con respecto al tema y ofreció respectivamente 2 y 3 razones de nivel 1 y subordinadas para la defensa de cada lado; por último, cerró con una conclusión. Después, en la figura 3 se muestra el resultado del segundo trabajo de composición textual de la alumna; en un primer vistazo, el esquema permite observar con claridad el crecimiento en la complejidad de la estructura general de su escrito, pues no sólo aumentó la cantidad total de elementos sino que además hizo uso de una mayor diversidad de elementos al incluir recursos como un punto de vista argumentativo, una refutación que estuvo respaldada por un grupo de razones y además utilizó marcadores 
funcionales como elementos de transición en la conformación de la cadena subordinada de razones para la refutación.

\section{COMENTARIOS FINALES}

Los propósitos de este documento consistieron en presentar una visión general del desempeño de los estudiantes con respecto a sus habilidades de argumentación escrita, es decir, conocer cómo los estudiantes universitarios construyen un texto argumentativo; y, además, reflexionar sobre el impacto que pudieran tener las intervenciones que se realizaron durante la segunda sesión de trabajo con el grupo de estudiantes. Dichos objetivos se lograron a través del análisis de los elementos que utilizan los estudiantes para construir los argumentos con los que defienden sus puntos de vista sobre un tema polémico.

Los resultados correspondientes a los textos que se elaboraron durante la primera sesión de trabajo, donde sólo se realizó una lectura antes de escribir, mostraron que los estudiantes en general incluyeron una cantidad moderada de elementos argumentativos funcionales totales en sus textos; pero la diversidad de recursos se mostró limitada, ya que mayormente los textos se conformaban tan sólo por la expresión del punto de vista y algunas razones para apoyarlo.

Por otra parte, en los textos resultantes de la segunda versión se mostró un aumento general tanto en la cantidad de elementos usados como en la diversidad de estos. Este avance se refleja principalmente en la inclusión de elementos como puntos de vista alternativos, contraargumentos y refutaciones, los cuales implican que hay cierto grado de conciencia sobre la existencia de una audiencia crítica que puede llegar a cuestionar y atacar el punto de vista personal del escritor; en una discusión oral esa audiencia está presente y la críticas se dan en el momento, tal como lo experimentaron los participantes en el aula al discutir el tema en grupo, pero al escribir, el interlocutor ya no está presente, por lo que es necesario adelantar esas posibles críticas y refutarlas para poder fortalecer la idea personal. Esto podría significar que las actividades de intervención realizadas en la segunda sesión de trabajo, tanto la discusión grupal como la exposición sobre el discurso argumentativo, pudieron haber tenido un impacto positivo en el desempeño de los estudiantes a pesar de la premura con la que se realizaron debido a las limitaciones de tiempo para trabajar con los estudiantes. En cuanto a esto, es probable que realizar las actividades con más tiempo arroje mejores resultados en la calidad de los textos, ya Linaloe León Baro doi: https://doi.org/10.36799/el.v5i1.102 Volumen 5, Número 1, Año 2020, ISSN: 2448-5942 
que podrían incluirse más actividades de formación y reforzamiento durante el proceso de elaboración de los mismos; sin embargo, para este trabajo no fue posible conseguir más sesiones de trabajo con los estudiantes, debido a que necesitaban continuar con sus cursos habituales, por lo tanto, fue preciso adaptar las actividades sólo a dos horas de trabajo.

También es necesario considerar que las expectativas sobre la cantidad y diversidad de elementos argumentativos no son el único factor determinante para una adecuada textualización de un discurso argumentativo (Chase, 2011). Pero, aunque no sea determinante la cantidad de elementos argumentativos para evaluar la calidad del texto, la inclusión de una diversidad amplia de elementos sí podría ser un factor de peso en la determinación de la solidez del texto argumentativo, pues las distintas funciones de cada elemento, en conjunto, permiten conformar defensas más sólidas para los puntos de vista. Por ejemplo, si además de razones se incluyen también elementos que consideran críticas posibles hacia el punto de vista que se defiende (puntos de vista alternativos o contraargumentos) y se refutan adecuadamente, puede lograrse una defensa más sólida para el punto de vista personal.

Claro que la descripción estructural de los argumentos es tan sólo un paso en el estudio de las habilidades de escritura argumentativa de los estudiantes universitarios, pues tal como mencionan Van Eemeren, et al (2006), además de las estructuras, es necesario también estudiar la solidez de la argumentación a partir del análisis del contenido de los argumentos, es decir, determinar la aceptabilidad de los argumentos empleados, dado que en ocasiones en el discurso argumentativo pueden presentarse inconsistencias lógicas o pragmáticas que lo vuelven débil. También es importante analizar aspectos textuales tanto a nivel lingǘstico como estructural en términos de coherencia y cohesión, entre muchos otros factores de la producción de textos que podrían tener influencia en la calidad global de un texto argumentativo; sin embargo, abordar el análisis de todos estos puntos en un solo estudio implicaría una complejidad de gran magnitud. Pero, gracias a las aportaciones de las distintas investigaciones que se citan en este documento y las que este mismo trabajo puedan generar, es posible crear un panorama general de las habilidades de argumentación escrita de los estudiantes universitarios, y además contribuyen a definir el camino a seguir para enriquecer aún más los conocimientos y propuestas dentro de esta área de estudio.

Linaloe León Baro doi: https://doi.org/10.36799/el.v5i1.102 Volumen 5, Número 1, Año 2020, ISSN: 2448-5942 
Por último, es importante destacar que tanto esta como otras investigaciones, aunque tengan distintas formas de abordar el análisis del desempeño de argumentación escrita de los estudiantes, todas convergen en la intención común de fomentar con mayor énfasis la práctica de la escritura argumentativa en las aulas universitarias, y de motivar a los estudiantes a ser más propositivos en la evaluación y confrontación de ideas, en lugar de sólo limitarlos a exponer y repetir contenidos. Para lograr esto es primordial que los expertos y los docentes en general asuman la tarea de crear más propuestas de intervención y estrategias didácticas que tengan la argumentación como eje de los procesos de enseñanza y aprendizaje durante la formación académica, dado que la práctica y reflexión constante sobre estas actividades representarían la base para que los alumnos adquieran mayor consciencia sobre la complejidad de la escritura y la argumentación, y lo fundamentales que son estas herramientas para la apropiación y creación de conocimientos (Castro y Sánchez, 2013; Bañalez Faz, et al, 2015). Así, al lograr convertirse en reguladores activos de sus propios procesos de análisis crítico, de escritura y de aprendizaje, podrán obtener resultados más satisfactorios en sus actividades como participantes del contexto disciplinar y profesional al que pertenezcan.

\section{IBLIOGRAFÍA}

Álvarez Angulo, T. y Ramírez Bravo, R. "Teorías o modelos de producción de textos en la enseñanza y aprendizaje de la escritura". Didáctica. Lengua y literatura. 18 (2006): 29-60. Recuperado de https://revistas.ucm.es/index.php/DIDA/article/view/DIDA0606110029A/19144

Andrade Calderón, M. C. "La escritura y los universitarios". Universitas Humanística, 68 (2009): 297-340. Recuperado https://revistas.javeriana.edu.co/index.php/univhumanistica/article/view/2277/1587

Bañalez Faz, Gerardo; Norma Alicia, Vega López; Narciso, Araujo Alvineda; Antonio Reyna Valladares y Brianda Saraí, Rodríguez Zamarripa. "La enseñanza de la argumentación escrita en la universidad”. Revista Mexicana de Investigación Educativa, 20 (2015): 879-910. Recuperado http://www.comie.org.mx/documentos/rmie/v20/n066/pdf/66008.pdf

Björk, Lennart y Ingegerd Blomstrand. La escritura en la enseñanza secundaria. Barcelona: Graó, 1994.

Calsamiglia, Helena y Amparo Tusón. Las cosas del decir: manual de análisis del discurso. Barcelona: Ariel, 2004.

Linaloe León Baro doi: https://doi.org/10.36799/el.v5i1.102 Volumen 5, Número 1, Año 2020, ISSN: 2448-5942 
Carlino, Paula. "Alfabetización académica: un cambio necesario, algunas alternativas posibles". Educere Investigación. 6. 20 (2003): 409-420.

Carlino, Paula. Escribir, leer y aprender en la universidad: Una introducción a la alfabetización académica. Buenos Aires: Fondo de Cultura Económica, 2005.

Cassany, Daniel. Describir el escribir: cómo se aprende a escribir. Barcelona: Paidós, 1989.

Cassany, Daniel. Construir la escritura. Barcelona: Paidós, 1999.

Castro Azuara, María Cristina y Martín Sánchez Camargo. "La expresión de opinión en textos académicos escritos por estudiantes universitarios". Revista Mexicana de Investigación Educativa. $\quad 18.57 \quad$ (2013): 483-506. Recuperado de http://www.comie.org.mx/revista/v2018/rmie/index.php/nrmie/article/view/255/2 $\underline{55}$

Chase, Beth Jillian. An Analysis of Argumentative Writing Skills of Academically Underprepared College Students. Tesis de Doctorado en Filosofía, Universidad de Columbia, 2011. Recuperado de https://pdfs.semanticscholar.org/c078/f26a75a01c0eb27606fa0be54e4ecd347578.pdf

Freedman, S. W. “Writing Instruction”. En B. Spolsky (Ed.), Concise Encyclopedia of Educational Linguistics. Oxford: Elsevier. (1999): 481-482.

Felton, Mark y Susanne Herko, "From dialogue to two-sided argument: Scaffolding adolescents' persuasive writing”. Journal of Adolescent and Adult Literacy. 47.8 (2004): 672-683. Recuperado de https://www.researchgate.net/publication/266477242 From dialogue to twosided argument Scaffolding adolescents' persuasive writing

Ferreti, Ralph; Lewis, William y Scott Andrews-Weckerly. "Do Goals Affect the Structure of Students' Argumentative Writing Strategies?” Journal of Educational Psychology, 101.3 (2009): 577-589. Recuperado de https://pdfs.semanticscholar.org/5bff/969c562794cf476e87766eb6d61e7d190524.pdf

Morales, Oscar Alberto y Norelkys Espinoza. "El desarrollo de la escritura de estudiantes universitarios". Lectura y Vida. (2005): 26-37.

Panahandeh, Esmaeil y Shahram Esfandiari, S. "The Effect of Planning and Monitoring as Metacognitive Strategies on Iranian EFL Learners' Argumentative Writing Accuracy". Procedia - Social and Behavioral Sciences, 98 (2014): 1409-1416. https://doi.org/10.1016/j.sbspro.2014.03.559 
Rodríguez Bello, Luisa Isabel. "El modelo argumentativo de Toulmin en la escritura de artículos de investigación educativa”. Revista digital universitaria, 5.1 (2004).

Van Eemeren, Frans, Peter Houtlosser y A.F Snoeck Henkemans. Argumentative Indicators in Discourse: A Pragma-Dialectical Study. The Netherlands: Springer, 2007.

Van Eemeren, Frans; Rob, Grootendorst; Francisca Henkemans y Roberto Marafioti. Argumentación: análisis, evaluación, presentación. Buenos Aires: Biblos, 2006.

Van Eemeren, Frans; Rob, Grootendorst. A Systematic Theory of Argumentation: The pagma-dialectical approach. Cambrigde: Cambridge University Pres, 2004. 